\title{
Acknowledgement to Reviewers of Forests in 2018
}

Forests Editorial Office

MDPI, St. Alban-Anlage 66, 4052 Basel, Switzerland

Published: 14 January 2019

Rigorous peer-review is the corner-stone of high-quality academic publishing. The editorial team greatly appreciates the reviewers who contributed their knowledge and expertise to the journal's editorial process over the past 12 months. In 2018, a total of 786 papers were published in the journal, with a median time to first decision of 17 days and a median time to publication of 42 days. The editors would like to express their sincere gratitude to the following reviewers for their cooperation and dedication in 2018:

Abbas, Dalia

Acosta, María Montserrat

Adhikari, Hari

Ågren, Göran I.

Agüera Vega, Francisco

Aguirre-Gutiérrez, Jesús

Ahmed, Iftekhar

Aizawa, Mineaki

Akamani, Kofi

Al-Ahmad, Hani

Alamgir, Mohammed

Albert, Matthias

Albrecht, Moritz

Albrechtová, Jana

Aleksandrowicz-Trzcińska, Marta

Aleta, Neus

Alfranca, Oscar

Alía, Ricardo

Alizoti, Paraskevi

Alkowni, Raed

Allen, Kathryn

Allen, Samuel

Almas, Andrew

Almendro-Candel, María Belén

Alvarez Taboada, Flor

Alvarez, Juan Gabriel

Álvarez-Álvarez, Pedro

Amaral Paulo, Joana

Ametzaga, Ibone

Amishev, Dzhamal

An, Hyunjin

Anadón, José D.

Ananda, Jayanath

Anderbrant, Olle
Anderson, Christopher

Anderson, James

Andivia, Enrique

Andreo, Veronica

Andrew, Carrie J.

Andronache, Ion C.

Aneece, Itiya $P$.

Antin, Cecile

Antonarakis, Alexander

Antunes, Cristina

Anyomi, Kenneth

Aparecido, Luiza Maria Teophilo

Aranda, Ismael

Aranha, Jose

Araus, Jose Luis

ArchMiller, Althea A.

Arévalo, José Ramón

Arief, Indrasumunar

Arjasakusuma, Sanjiwana

Arriga, Nicola

Arts, Koen

Aruga, Kazuhiro

Asrar, Ghassem R.

Assal, Timothy

Atkins, Jeff

Aubry-Kientz, Melaine

Augenstein, Isabel

Avalos, Gerardo

Averill, Kristine

Axelson, Jodi B.

Ayrey, Elias

Azouzi, Riadh

Babcock, Chad

Babcock, Roger 
Baczek-Kwinta, Renata

Bader, Maaike

Bāders, Endijs

Baker, Thomas P.

Bakker, Mark Ronald

Balenović, Ivan

Baležentienè, Ligita

Ballhorn, Daniel J.

Balogh-Brunstad, Zsuzsanna

Balsi, Marco

Banaszuk, Piotr

Baral, Sony

Barbati, Anna

Barber, Paul

Barbosa Da Silva, Evandro

Barchi, Lorenzo

Barrio-Anta, Marcos

Barriocanal, Carles

Barros, Ana M. G.

Barsberg, Søren Talbro

Bartlow, Andrew W.

Bascietto, Marco

Battisti, Corrado

Bauch, Martin

Bauman, Jenise

Bauwens, Sebastien

Baynes, Jack

Bayrak, Mucahid

Beadle, Chris

Beguin, Julien

Behnisch, Martin

Belart, Francisca

Belbo, Helmer

Belgiu, Mariana

Bélisle, Annie Claude

Bell, F. Wayne

Bell, Simon

Bellingham, Peter

Belloncle, Christophe

Belval, Erin J.

Bembenek, Mariusz

Bemmels, Jordan B.

Bento-Gonçalves, António

Benton, Elizabeth

Berg, Björn

Berger, Ambros

Bergström, Dan

Bernardo, Umberto

Berrill, John-Pascal

Berta, Graziella

Bertocci, Michele

Bertomeu, Mercedes
Berveglieri, Adilson

Bęś, Agnieszka

Betti, Marco

Bezos, Diana

Bhandari, Santosh

Bhardwaj, Anshuman

Bidel, Luc

Bielinis, Ernest

Bigelow, Seth

Bijak, Szymon

Bilodeau-Gauthier, Simon

Birhane, Emiru

Biziks, Vladimirs

Blair, Patricia

Blanc, Simone

Blanc-Jolivet, Céline

Blank, Robert

Blankinship, Joseph C.

Blaschke, Paul

Błaszczak-Bąk, Wioleta

Błońska, Ewa

Bloomfield, Keith

Bluffstone, Randall

Bobiec, Andrzej

Bogdanski, Bryan

Bogdziewicz, Michał

Bolibok-Bragoszewska, Hanna

Boltiziar, Martin

Bolton, Nicholas

Bonachea, Jaime

Bonari, Gianmaria

Bond-Lamberty, Ben

Bonga, Jan Max

Booth, Trevor

Boroń, Piotr

Borůvka, Vlastimil

Boscutti, Francesco

Bose, Arun Kanti

Bouchard, Mathieu

Boucher, Étienne

Bourgeois, Bérenger

Boyce, Richard

Bracho, Rosvel

Brack, Cristopher

Bragg, Don

Brandle, James R.

Bråthen, Kari Anne

Breinig, Lorenz

Breschan, Jochen

Bretfeld, Mario

Brienen, Roel J. W.

Brischke, Christian 
Brockhaus, maria

Brokaw, Nicholas

Broll, Gabriele

Bronisz, Karol

Brouwers, Niels

Brovkina, Olga

Brown, Nathan

Brunn, Melanie

Brzeziecki, Bogdan

Bucharova, Anna

Buchholz, Thomas

Bunn, Andy

Burivalova, Zuzana

Burke, Jordan L.

Burkhardt, Jürgen

Burkhart, Harold E.

Burns, Kelly S.

Burns, Sarah L.

Burton, Andrew J.

Burton, Julia

Buse, Jörn

Bushakra, Jill M.

Busse, Matt

Butler, William

Bygaart, A. J. Vanden

Cabo, Carlos

Cadman, Timothy

Cai, Zhen

Cale, Jonathan

Calef, Monika P.

Calfapietra, Carlo

Calvo, Leonor

Camenzind, Tessa

Campagnaro, Thomas

Campbell, Robert M.

Campos-Taberner, Manuel

Cañas, Rafael A.

Candelier, Kévin

Cannon, Chuck

Cannon, Jeffery B.

Cano, Jose Antonio Navarro

Cao, Lin

Caputo, Jesse

Carl, Christin

Carmona-Moreno, Cesar

Carodenuto, Sophia

Carr, Craig

Carrer, Marco

Casals, Pere

Case, Bradley

Caspersen, John

Catalin Petritan, Ion
Cater, Matjaz

Cavender-Bares, Jeannine M.

Cavero, José

Cazzato, Eugenio

Cedamon, Edwin

Chalivendra, Subbaiah C.

Chalot, Michel

Chang, Scott $X$.

Chapman, Mark

Charles, Lachlan

Charvátová, Hana

Chatterjee, Amit

Cheeseman, John M.

Chen, Shih-Yu

Chen, Yuwei

Chendev, Yury

Chhin, Sophan

Chianucci, Francesco

Chiavetta, Ugo

Chiba, Yukihiro

Chimungu, Joseph

Chmura, Damian

Chojnacka-Ożga, Longina

Chojnacky, David

Chong, Geneva W.

Christe, Camille

Christina, Mathias

Christoffersen, Bradley

Christoph, Rupprecht

Ciobotaru, Ana-Maria

Ciolli, Marco

Clark, Kenneth

Cleaver, Christy M.

Clivot, Hugues

Cloy, Joanna

Coble, Dean

Cockfield, Geoff

Cocozza, Claudia

Coen, Janice L.

Coleman, Mark

Colfer, Carol

Colin Koeniguer, Elise

Combe, Marie

Comeau, Phillip G.

Conner, William H.

Conrad, Anna

Cook, Stephen P.

Cordonnier, Thomas

Corona, Piermaria

Correia, Barbara Dos Santos

Correia, Marisol

Corrigan, Edwin 
Costafreda-Aumedes, Sergi

Costanza, Kara

Coutinho, Teresa

Crain, Brent

Crandall, Raelene

Creutzig, Felix

Cridge, Andrew

Cubry, Philippe

Cumming, Jonathan

Curtis, Peter

Cvitanovic, Marin

Cvrčková, Helena

D'Acci, Luca

Daebeler, Anne

Dahle, Gregory

Dahlen, Joseph

Dai, Shu-Mei

Dale, Virginia

D'Amore, David V.

D'Andrea, Ettore

Daniel, Nadal-Sala

Danson, Mark

Darbyshire, Robyn

Dařenová, Eva

Das Gupta, Sanatan

Dass, Pawlok

Davies, Althea

Davis, Joshua C.

Davydenko, Kateryna

Dawson, Wayne

Dawson-Andoh, Benjamin E.

De Caceres, Miquel

De Cinti, Bruno

De Dios, Víctor Resco

De Francesco, Fabio

De Grandi, Elsa Carla

De Jong, Wil

De Lafontaine, Guillaume

De Meo, Isabella

De Miguel, Sergio

De Mil, Tom

De Toro, Pasquale

Deane-Coe, Kirsten

Dee, Justin R.

Dees, Matthias

Defo, Maurice

Dehnen-Schmutz, Katharina

Delgado, Juan Antonio

Delshammar, Tim

Demertzi, Martha

Denisow, Bozena

Deo, Ram K.
DesRochers, Annie

DeWalt, Saara

Dhital, Narayan

Di Lallo, Giulio

Diaci, Jurij

Díaz-Balteiro, Luis

Diaz-Barradas, Mari Cruz

Diaz-Hernandez, Jose Luis

Didion, Markus

Diiwu, John

Dimitrakopoulos, Panayiotis G.

Dinulică, Florin

Dirnböck, Thomas

Disney, Mathias

Dlapa, Pavel

Dobre, Mariana

Dobrowolska, Dorota

Đodan, Martina

Doetterl, Sebastian

Dolan, Benjamin

Domec, Jean-Christophe

Dong, Chun-Yu

D'Orangeville, Loïc

Dostálek, Tomáš

Doussan, Claude

Dreyer, Erwin

Drosos, Vasileios C.

Drouet, Thomas

Drozdowski, Stanisław

Duan, Jian

Duane, Andrea

Ducey, Mark J.

Dudits, Dénes

Duduman, Gabriel

Duff, Thomas

Đuka, Andreja

Dunn, Christopher

Dutschke, Michael

Dvořák, Jiří

Dvořák, Miloň

Dwivedi, Puneet

Dyderski, Marcin K.

Dye, Alex W.

Dymond, Salli F.

Edwards, Christy

Egerton-Warburton, Louise

Egertsdotter, Ulrika

Ehbrecht, Martin

Eid, Tron

Einzmann, Helena JR

Eliades, Marinos

Eller, Cleiton 
Elsa, Varela

Elsasser, Peter

Elsayed, Saber M.

Emery, Marla

Enderle, Rasmus

Ennos, Richard

Ersson, Back Tomas

Esbrí, José María

Escamez, Sacha

Essien, Charles

Etzold, Sophia

Evans, David

Evenden, Maya L.

Ezell, Andrew W.

Facciotto, Gianni

Fagerstedt, Kurt

Falistocco, Egizia

Fanin, Nicolas

Fauset, Sophie

Feikema, P. M.

Ferfuia, Claudio

Fernandes, João

Fernández, Manuel

Fernández-Manso, Alfonso

Fernández-Martínez, Marcos

Fernandez-Ondoño, Emilia

Ferrenberg, Scott

Ferrio, Juan Pedro

Ferster, Colin

Fetcher, Ned

Feurer, Melanie

Ficko, Andrej

Field, Richard

Fields, Paul

Filipescu, Cosmin

Finckh, Manfred

Finer, John J.

Fioretto, A.

Fioretto, Antonietta

Flather, Curtis H.

Floistad, Inger Sundheim

Floris, Antonio

Flower, Charles

Flower, Charles E.

Fonseca, Teresa

Foote, Julie

Foote, Lee

Fornwalt, Paula J.

Forrester, Jodi

Forster, Michael A.

Forsthuber, Boris

Fortin, Mathieu
Foster, Adrianna

Foster, Alec

Fotis, Alexander

Frampton, John

Francesco, Negro

Francini, Gaia

Franklin, Jennifer

Franklin, Rima B.

Fraser, Jacob

Fratini, Roberto

Fraver, Shawn

Fredericksen, Todd

Freese, Dirk

Frelich, Lee

French, Nancy

Freppaz, Michele

Frey, Gregory E.

Friend, Andrew D.

Fuentealba, Alvaro

Fuentes, Mauricio

Fulvio Ducci, Fulvio

Galiatsatos, Nikolaos

Galko, Juraj

Gallagher, Tom

Gallemore, Caleb

Galos, Borbala

Gamarra, Javier G. P.

Ganatsas, Petros

Ganzhorn, SM

Garcia-Franco, Noelia

Garcia-Haro, Javier

Gautam, Shuva

Gauthier, Martin-Michel

Gautreau, Pierre

Gazda, Anna

Gélinas, Nancy

Germain, René

Getzner, Michael

Ghanem, Michel Edmond

Gharun, Mana

Giannetti, Francesca

Giannico, Vincenzo

Giergiczny, Marek

Gilland, Keith E.

Gil-Pelegrín, Eustaquio

Giordano, Luana

Giraldo, Ramón

Girona, Miguel Montoro

Giuliani, Felice

Glick, Henry

Goebel, Charles

Goeking, Sara A. 
Goldfarb, Barry

Goldingay, Ross

Goldmann, Kezia

Gómez-Brandón, María

Gömöry, Dušan

Gömöryová, Erika

Gonzalez, Ronalds

González-Martínez, Santiago C.

Gorchov, David

GORIŠEK, Željko

Gouker, Fred

Grabarnik, Pavel

Gradel, Alexander

Graham, Eric

Granhus, Aksel

Graves, Sarah J.

Gray, Andrew

Gray, Gordon R.

Greaves, Heather

Greenslade, Penelope

Gregoire, Timothy

Grigolato, Stefano

Grinsven, Matthew Van

Groninger, John

Grootemaat, Saskia

Gross, Cole D.

Großmann, Josef

Grossnickle, Steven C.

Grulová, Daniela

Gruselle, Marie-Cecile

Gu, Huan

Guangyu, Wang

Guccione, Pietro

Guidi Nissim, Werther

Guillemette, François

Guiterman, Christopher

Güney, Aylin

Gunn, John

Guo, Qinghua

Gustafson, Eric J.

Guyassa, Etefa

Guzmán-Delgado, Paula

Gwenzi, David

Haara, Arto

Haase, Diane

Häbel, Henrike

Hadziabdic, Denita

Hagan, Donald L.

Haines, Heather

Haire, Sandra

Hakkenberg, Christopher

Hale, Sophie
HALIK, Ümüt

Halvorsen, Kathleen

Hamberg, Leena

Hamerlynck, Erik P.

Hamraz, Hamid

Hanba, Yuko T.

Hanberry, Brice

Hanft, Kim Littke

Hanley, Nicholas

Hansen, Andrew

Hansen, Endre Hofstad

Hardy, Giles

Harkess, Richard

Harmon, Mark

Hartmann, P.

Harwood, Christopher

Hasenauer, Hubert

Hatala Matthes, Jaclyn

Haughian, Sean R.

Haugo, Ryan

Hawryło, Paweł

Hayashi, Takahisa

Hayes, Daniel

He, Liming

$\mathrm{He}$, Minhui

Headlee, William L.

Heartsill Scalley, Tamara

Hedley, Richard

Heibl, Christoph

Hein, Jonas

Helman, David

Hember, Robbie

Hember, Robbie A.

Henders, Sabine

Hennessy, Logan A

Herman, Krzysztof

Herms, Dan

Hernandez, Alexander

Hernik, Józef

Herrero, Asier

Herrero, Hannah

Herrero, Manuel Souto

Herschbach, Cornelia

Hesser, Franziska

Hidalgo, Pablo J.

Hiesl, Patrick

Hietala, Ari M.

Hill, David J.

Hilszczańska, Dorota

Hinesley, Eric

Hinesley, L. Eric

Hirata, Yasumasa 
Hiromi, Mizunaga

Hirschmugl, Manuela

Hladnik, David

Hodel, Richard G.J.

Hoen, Hans

Hof, Anouschka R.

Hoffmann, Stephan

Hofhansl, Florian

Hogan, J. Aaron

Högbom, Lars

Holder, Curtis D.

Holmgren, Sara

Holmström, Emma

Holzleitner, Franz

Hopkins, Anna

Hořák, David

Horemans, Joanna

Hossain, Mohammad Shawkat

Houdet, Joël

Hrašovec, Boris

Hsieh, Chi-Ming

Huang, Jr-chuan

Huang, Meng-Yuan

Huang, Wenjuan

Hubbard, William

Hudek, Csilla

Hudzieczek, Vojtech

Huebner, Cynthia

Hughes, M. Joseph

Huhta, Esa

Hujala, Teppo

Humagain, Kamal

Hunt, Harriet V

Huq, Nazmul

Hynynen, Jari

Hytonen, Jyrki

Ibanez, Thomas

Idol, Travis

Ievinsh, Gederts

Iiames, John

Iizuka, Kotaro

Imbert Rodríguez, Juan Bosco

Imeson, Anton

Ingwers, Miles W.

Ingwersen, Joachim

Ioos, Renaud

Ioras, Florin

Irga, Peter

Irland, Lloyd

Irulappa Pillai Vijayakumar, Dinesh Babu

Ivanov, Yury

Iwaizumi, Masakazu G
Jabran, Khawar

Jacobi, William

Jagodzinski, Andrzej

Jamilena, Manuel

Jandl, Robert

Jang, Woongsoon

Janga, Madhusudhana

Jánosi, Imre M.

Jaung, Wanggi

Jayathunga, Sadeepa

Jenkins, Michael

Jepsen, Jane Uhd

Jien, Zhang

Jin, Hongxiao

Jo, Insu

John, Bailey

Johnsen, Kurt

Johnson, Adelaide C.

Johnson, Brittany

Johnson, Daniel J.

Johnson, Jeremy

Johnstone, Denise

Jomura, Mayuko

Jones, Dryw A.

Jones, Ian

Jones, Michael

Jordan, Marie Odile

Jules, Erik S.

Julich, Dorit

Jung, Christopher

Jurkšienè, Girmantè

Kabała, Cezary

Kacálek, Dušan

Kacaniova, Miroslava

Kačik, František

Kagawa, Takahide

Kainer, David

Kaitaniemi, Pekka

Kajimoto, Takuya

Kalliovirta, Leena

Kalsi, Megha

Kalusova, Veronika

Kaminaka, Hironori

Kamperidou, Vasiliki

Kane, Michael

Kanerva, Sanna

Kangas, Annika

Kanowski, Peter J.

Kant, Shashi

Kanzian, Christian

Kappler, Rachel H.

Karaszewski, Zbigniew 
Kärhä, Kalle

Karsenty, Alain

Karttunen, Kalle

Kasanen, Risto

Kasel, Sabine

Kashian, Dan

Kasischke, Eric

Kašpar, Jan

Kasprzyk, Idalia

Kautz, Markus

Kawa, Arkadiusz

Kayama, Masazumi

Kazana, Vassiliki

Kearsley, Elizabeth

Keller, Elizabeth

Kelsey, Katharine

Kenfack, David

Keren, Srđan

Kerhoulas, Lucy

Kershaw, John

Keskitalo, Eva Carina Helena

Keyser, Tara

Khadempour, Lily

Khechine, Hager

Kilgore, Jason

Kim, Dohyoung

Kim, Hyunseok

Kim, Jae Su

Kim, Jae-Jin

Kim, Jinwon

Kim, Kee Dae

Kimsey, Mark

Kincaid, Joshua A.

Kirst, Henning

KITAO, Mitsutoshi

Kitaoka, Satoshi

Kivimäenpää, Minna

Kizha, Anil

Kjønaas, O. Janne

Klavina, Darta

Klein, Tamir

Klemmt, Hans-Joachim

Klesse, Stefan

Klitkou, Antje

Klocko, Amy

Klopčič, Matija

Klutsch, Jennifer

Knapp, Benjamin O.

Knapp, Dániel G

Knapp, Nikolai

Knelman, Joseph

Knelman, Joseph E.
Knight, Kathleen

Knoke, Thomas

Knowles, John

Kobayashi, Natsuko I.

Koda, Eugeniusz

Koehler, Hartmut

Köhl, Michael

Koide, Roger T.

Kolb, Evelyne

Kolb, Thomas

Kong, Lisheng

Konôpka, Bohdan

Konrad, Heino

Koprowski, Marcin

Korecky, Jiri

Koreň, Milan

Korets, Mikhail

Kosiba, Alexandra

Koster, Kajar

Košulič, Ondřej

Kotowska, Martyna M.

Koulelis, Panagiotis

Koumba-Yoya, Georges

Koutoulis, Anthony

Kowalczyk, Jan

Koydemir, Hatice

Kozlov, Mikhail

Krajewski, Piotr

Kralik, Martin

Kramer, Heather Anu

Kranabetter, J. M.

Krause, Cornelia

Kregar, Klemen

Krejza, Jan

Krigstin, Sally G.

Kucbel, Stanislav

Kühmaier, Martin

Kumar Baul, Tarit

Kume, Atsushi

Kunz, Jörg

Kusbach, Antonín

Kutnar, Lado

Kuželka, Karel

Kvamme, Torstein

Kwiatkowska-Malina, Jolanta

Kwon, Hyojung

Kwon, Youngsang

La Mantia, Tommaso

Lacuna-Richman, Celeste

Lal, Pankaj

Lamarche, Josyanne

Lamersdorf, Norbert 
Landgren, Chal

Landuyt, Dries

Laneve, Giovanni

Lanfredi, Maria

Lang, Ashley

Lang, Friederike

Lang, Ingeborg

Lange, Holger

Langholtz, Matthew

Langner, Andreas

Lapointe, Stephen

Laratte, Bertrand

Laroche, Genevieve

Larsen, Chris

Larsen, David R.

Larson, Evan R

Latifi, Hooman

Lavergne, Aliénor

Lawson, Shaneka S

Lazár, Dušan

Lazdina, Dagnija

Le Tacon, Francois

Leberecht, Martin

LeDuc, Stephen D.

Lee, Peter Sang-Hoon

Lee, Seung-Il

Lee, Thomas D.

Lee, Woo-Kyun

Legge, Allan

Lensing, Janet

Leppänen, Jussi

Lesniewska, Feja

Letcher, Susan G.

Leverkus, Alexandro B.

Levinsson, Anna

Lewis, James D.

Lhotka, John

Li, Yun

Liang, Haiying

Liao, Pei-Chun

Liechty, Hal O.

Lieffers, Victor

Liimatainen, Maarit

Limousin, Jean-Marc

Lin, Tengchiu

Linaldeddu, Benedetto

Lindahl, Karin Beland

Lindner, André

Linnakoski, Riikka

Lintunen, Jussi

Liu, Chao-Lin

Liu, Houping
Liu, Ning

Liu, Weibo

Llovería, Raquel Montorio

Locky, David

Lojka, Bohdan

Lombardi, Fabio

Lombardozzi, Danica

Long, James

Longworth, J. Benjamin

Looney, Christopher E.

Lopatin, Javier

Lopez C., M. Larry

López De Heredia, Unai

Lopez, Bianca

Lopez-Angulo, Jesus

López-Tirado, Javier

Losada Sánchez, María Nieves

Loudermilk, Louise

Louman, Bas

Lovric, Marko

Lozano-Parra, Javier

Lstibůrek, Milan

Lu, Dau-Jye

$\mathrm{Lu}$, Meng

Luc, Malgorzata

Lucash, Melissa S.

Lúcia Gonçalves, Ana

Luetz, Cornelius

Lugo, Ariel

Lujan-Alvarez, Concepcion

Lumibao, Candice

Lundén, Karl

Lundqvist, Lars

Lykidis, Charalampos

Lynch, Thomas B.

$\mathrm{Ma}, \mathrm{Wu}$

Macek, Petr

Machado, Rui

Macinnis-Ng, Cate

Macquarrie, Chris J. K.

Madrigal, Javier

Maesano, Mauro

Magagnotti, Natascia

Maggard, Adam

Magnabosco Marra, Daniel

Magnussen, Steen

Mahan, Carolyn G.

Mahmood, Taufique

Mainwaring, Mark

Malanson, George P.

Malatak, Jan

Malhotra, Avni 
Malinen, Jukka

Malone, Sparkle L.

Maloney, Patricia

Man, Cosmin

Man, Rongzhou

Mansuy, Nicolas

Mantilla-Contreras, Jasmin

Maraseni, Tek

Marçais, Benoit

Marcella, Windmuller-Campione

Marchi, Maurizio

Marek, Michal

Måren, Inger

Maresi, Giorgio

Margolis, Ellis

Maricle, Brian R.

Marizzi, Christine

Marjanovic, Hrvoje

Marks, Christian

Marozas, Vitas

Marques, Alexandra

Marques, Susete

Marra, Daniel Magnabosco

Marshall, Peter L

Martin, Mokroš

Martin, Ruth

Martin-Benito, Darío

Martínez-Álvarez, Pablol

Martínez-Mena, María

Martínez-Sancho, Elisabet

Marusak, Robert

Marziliano, Pasquale A.

Masiero, Mauro

Massaccesi, Luisa

Matonis, Megan

Matsushita, Michinari

Matsuura, Toshiya

Matzek, Virginia

Mauro, Francisco

Mayfield, Helen

Mazziotta, Adriano

Mbatu, Richard S.

McClaugherty, Charles

McConnell, Thomas Eric

Mcdermid, Greg

McDermott, Shana

Mcewan, Ryan

McGroddy, Megan E

McNab, Henry

Méausoone, Pierre-Jean

Mederski, Piotr S.

Medved, Sergej
Meigs, Garrett

Mellmann-Brown, Sabine

Meloni, Fabio

Melzer, Rainer

Meng, Ran

Mercader, Rodrigo

Merganič, Ján

Mergelov, Nikita

Messier, Christian

Messinger, Max

Metslaid, Marek

Meunier, Félicien

Mi Sun, Park

Michalet, Richard

Mihai-Leonard, Duduman

Mikuła, Anna

Milenkovic, Milutin

Milios, Elias

Milotic, Tanja

Minckley, Thomas A.

Minocha, Subhash C.

Minor, Jesse

Miquel, Martine

Miranda, Carlos

Miranda, Jose Carlos

Miścicki, Stanisław

Mishra, Pawan Kumar

Misra, Gourav

Miura, Toshiko

Miyamoto, Kazuki

Modica, Marco

Mokros, Martin

Molina-Terrén, Domingo

Molowny-Horas, Roberto

Monaco, Thomas A.

Mongil-Manso, Jorge

Monleon, Vicente

Montagnoli, Antonio

Monteiro, Jose A.

Monteuuis, Olivier

Monteverdi, Maria Cristina

Montiel Molina, Cristina

Montwé, David

Morales, Narkis S

Morales-Barquero, Lucia

Morelli, Toni Lyn

Moreno-Sanchez, Rafael

Moriana, Rosana

Moricz, Norbert

Morita, Kanako

Morris, Jesse L.

Moser, Astrid 
Mosseler, Alex

Möttönen, Veikko

Moutahir, Hassane

Moya, Daniel

Mozgeris, Gintautas

Mróz, Anna

Muir, Jasmine

Mukhortova, Liudmila

Müller, Markus

Müllerová, Jana

Mullet, Martin

Munck, Isabel

Mund, Jan-Peter

Murphy, Stephen J.

Mustalahti, Irmeli

Mutanen, Antti

Myers, Wayne

Nabais, Cristina

Nadeau, Solange

Næsset, Erik

Nagamitsu, Teruyoshi

Nagel, Thomas A.

Nair, Richard

Nakada, Ryogo

Nakamura, Kazuhiko W.

Nanos, Nikos

Natascha Wagner, Natascha

Negron, Jose

Neinavaz, Elnaz

Nelson, Harry

Neophytou, Charalambos

Neudert, Regina

Neufeld, Howard

Neves Proença, Diogo

Newton, Peter

Newton, Peter F.

Neyland, Mark

Nichols, J. Doland

Niederman, Robert A.

Niemczyk, Marzena

Nieminen, Tiina $\mathrm{M}$.

Niemz, Peter

Niklas, Karl

Nilsen, Erik T

Ningthoujam, Ramesh

Nirola, Ramkrishna

Noborio, Kosuke

Nocetti, Michela

Noguchi, Kyotaro

Nolet, Philippe

Nophea, Sasaki

Novak, Klemen
Nugnes, Francesco

Numata, Izaya

Nunes, Adélia

O'Connor, Christopher D.

Obataya, Eiichi

Oberle, Brad

Obojes, Nikolaus

Ogawa, Kazuharu

Ogaya, Romà

Ohtsuka, Toshiyuki

Olano, José Miguel

Olivar, Jorge

Olson, Matt

Olsson, PerOla

Olsson, Sanna

Ondreičková, Katarína

Ongolo, Symphorien

Ono, Kenji

Ordóñez, Camilo

Orlova-Bienkowskaja, Marina J.

Orlowski, Natalie

Oses Eraso, Nuria

Osone, Yoko

Ostberg, Johan

Ostrogović Sever, Maša Zorana

Oszako, Tomasz

Oten, Kelly

Ots, Katri

Overby, Steven T.

Overdieck, Dieter

Overman, Han

Owari, Toshiaki

Pacaldo, Renato S.

Pach, Maciej

Pacheco, Pablo

Page-Dumroese, Deborah S.

Pagliarani, Chiara

Palaiologou, Palaiologos

Paleari, Michele

Paluš, Hubert

Pan, Pan

Panagiotidis, Dimitrios

Panagopoulos, Thomas

Pandur, Zdravko

Pandža Bajs, Irena

Pansing, Elizabeth

Panyushkina, Irina P.

Papa, Ivica

Paradis, Gregory

Paris, Pierluigi

Parmehr, Ebadat Ghanbari

Parobeková, Zuzana 
Parrotta, John

Partanen, Sari

Pasgaard, Maya

Pastorella, Fabio

Patočka, Zdeněk

Patterson, Thomas

Paudyal, Ramesh

Paungfoo-Lonhienne, Chanyarat

Pawłowski, Tomasz A.

Paynter, Ian

Pearman, Peter

Pec, Gregory

Pedersen, Ole

Peguero-Pina, José Javier

Peinado, Ricardo Ruiz Peinado

Peltola, Heli

Pelz, Kristen

Pérez-Campaña, Rocío

Perry, Kayla I.

Pescott, Oliver L.

Peterson, Birgit

Peterson, Chris J.

Petritan, Any Mary

Petropoulos, George

Peuhkurinen, Jussi

Pham, Tien Dat

Picard, Nicolas

Picchi, Gianni

Picciau, Rosangela

Pichler, Viliam

Pielech, Remigiusz

Piermattei, Alma

Piernik, Agnieszka

Pierskalla, Chad

Pierskalla, Chad D.

Piispanen, Riikka

Pile, Lauren

Pilli, Roberto

Pimentel, C. S.

Pinchot, Cornelia C.

Pinno, Brad

Pinzon, Jaime

Pittman, Jeremy

Płażek, Agnieszka

Plotkin, Audrey

Pniewski, Tomasz

Podrebarac, Frances A.

Pohjanmies, Tähti

Poje, Anton

Pokonieczny, Krzysztof

Poljak, Igor

Pollastrini, Martina
Polo-Gómez, María José

Ponce-Hernandez, Raul

Poosakkannu, Anbu

Porth, Ilga

Portillo-Estrada, Miguel

Poteri, Marja

Pothier, David

Potočnik, Igor

Potterf, Maria

Potts, Matthew

Pourmokhtarian, Afshin

Poursanidis, Dimitris

Powers, Matthew

Preisler, Haiganoush K.

Priess, Jörg

Prislan, Peter

Prisley, Steve

Przybysz, Arkadiusz

Puettmann, Klaus

Puliti, Stefano

Pulkkinen, Pertti

Quesada, Tania

Quijano, Laura

Quintano, Carmen

Rademacher, Peter

Radu-Daniel, Pintilii

Rahlf, Johannes

Rahman, Mizanur

Rahman, Mohammad A

Raich, James W.

Rambal, Serge

Ramcilovik-Suominen, Sabaheta

Rammer, Werner

RamoBarrena, Ramo

Ranger, Christopher

Rasi, Rastislav

Räty, Minna

Raub, Florian

Raymond, Jay E.

Rebek, Eric J.

Redeker, Kelly

Reding, Michael

Redman, Charles

Reid, Anya

Reid, Doug

Reilly, Matt

Reis, Carlos M. G.

Rembiałkowska, Ewa

Remeš, Jiří

Renninger, Heidi

Resco, Víctor

Revermann, Rasmus 
Richard, Sniezko

Riesco Munoz, Guillermo

Rigling, Daniel

Rigolon, Alessandro

Rigsby, Chad Michael

Rikiishi, Kazuhide

Rita, Angelo

Ritter, Tim

Ro, Hee-Myong

Robert, Christelle

Roberts, David

Roberts, Scott

Robeson, Scott M.

Robinson, Kathryn M.

Rochefort, Regina

Rock, Joachim

Rodrigues, Marcos

Rodriguez, Georgina

Roe, Amanda D.

Rogeau, Marie-Pierre

Roig Gomez, Sonia

Rollinson, Christine

Romeralo, Carmen

Romero, Claudia

Rönnqvist, Mikael

Roscoe, Lucas E.

Rossbach, Silvia

Rowe, Brad

Rudemo, Mats

Rudrabhatla, Sairam

Rugman-Jones, Paul

Ruiz-González, Ana Daría

Ruiz-Yanetti, S.

Runkle, James R.

Rusterholz, Hans-peter

Rutishauser, Ervan

Ryall, Krista

Rydzkowski, Tomasz

Rylott, Elizabeth L

S. Dodd, Richard

Sá, Ana C. L.

Saarela, Svetlana

Saarinen, Ninni

Sačkov, Ivan

Sah, Jay P.

Saha, Somidh

Saito, Yoko

Saiz-Fernández, Iñigo

Saksa, Timo

Saladyga, Thomas

Salata, Stefano

Salbitano, Fabio
Salle, Aurelien

Salom, Scott

Sample, V. Alaric

Sánchez-Pinillos, Martina

Sangireddy, Sasikiran Reddy

Santopuoli, Giovanni

Sanz-Saez, Alvaro

Sato, Hisashi

Sattley, W. Matthew

Saud, Pradip

Savi, Tadeja

Savvides, Andreas

Scalenghe, Riccardo

Schepaschenko, Dmitry

Scherrer, Daniel

Schindlbacher, Andreas

Schindler, Stefan

Schlesinger, William

Schlund, Michael

Schmerbeck, Joachim

Schmidt, Isabel B.

Schmitt, Christine B.

Schowalter, Timothy

Schultz, Courtney

Schulz, Tobias

Schulze, Ernst-detlef

Schumacher, Johannes

Schuster, Michael

Schweier, Janine

Schweigkofler, Wolfgang

Schweizer, Pia-Johanna

Scotti, Roberto

Scullion, John

Sebastiani, Federico

Šebeň, Vladimír

Seidel, Dominik

Seielstad, Carl

Seijo, Francisco

Seiler, John

Selas, Vidar

Semenov, Mikhail V.

Semmens, Darius

Sena, Kenton

Senf, Cornelius

Sevillano, Ignacio

Seyfferth, Carolin

Sferlazza, Sebastiano

Shahi, Chander

Shahzad, Muhammad

Shao, Gang

Shao, Guofan

Shao, Xiaowei 
Shapiro, Aurélie

Sharma, Ram P.

Shepherd, Brenda

Sheppard, Jonathan

Sheridan, Gary

Shiau, Yo-Jin

Shin, Won Sop

Shinohara, Yoshinori

Showalter, David

Shrestha, Paliza

Shrestha, Santosh

Sieg, Carolyn

Siegert, Courtney

Siemann, Evan

Sierra, José Ramón Arévalo

Sigmund-Schultze, Marianna

Siljanen, Henri M.P.

Sills, Erin

Silva, Carlos Alberto

Silva, Luís

Sirca, Costantino

Sitzia, Tommaso

Škorpík, Petr

Skre, Oddvar

Skutsch, Margaret

Sladonja, Barbara

Slavoff, Sarah A

Śliwińska-Wilczewska, Sylwia

Smagin, Andrey Valentinovich

Smidt, Mathew

Smith, James

Smith, Steven M.

Snell, Rebecca S

Snook, Laura

Socha, Jarosław

Sokolov, Anton

Solla, Alejandro

Solomou, Alexandra

Soolanayakanahally, Raju

Soto-Berelov, Mariela

Sousa, Vicelina B.

Sousa-Silva, Rita

Spinelli, Raffaele

Spyroglou, Gavriil

Stahlheber, Karen A.

Stängle, Stefan

Stanton, Daniel E.

Stares, Mitchell G.

Steenackers, Marijke

Steffan, Joshua J.

Stein, Taylor V.

Steiner, Kim
Stejskal, Jan

Stelinski, Lukasz

Stenberg, Leena

Sterba, Hubert

Stevens, M. Henry H.

Stevens, Michael T.

Stevens-Rumann, Camille

Stewart, Jane

Štochlová, Petra

Stoehr, Michael

Storer, Andrew

Stout, Susan L.

Stovall, Atticus

Strack, Maria

Strandgard, Martin

Straže, Aleš

Strigul, Nikolay

Strimbu, Bogdan

Strimbu, Vlad C.

Strukelj, Manuella

Strunk, Jacob L.

Stutz, Kenton P.

Subervie, Julie

Sugimoto, Tomoko

Sukdeo, Nicole

Sułkowska, Małgorzata

Sullivan, Andrew

Sullivan, Benjamin

Sun, Wu

Suontama, Mari

Surovy, Peter

Susaeta, Andres

Svensson, Johan

Sweeney, Jon

Synowiec, Agnieszka

Szmidt, Alfred E.

Szmyt, Janusz

Szulecka, Julia

Szwagrzyk, Jerzy

Taiki, Mori

Takahashi, Misa

Takao, Gen

Talbot, Bruce

Talhelm, Alan F.

Tallant, J.

Tampekis, Stergios

Tamura, Atsushi

Tan, Zhou

Tananaev, Nikita

Tanger, Shaun

Tanner, Edmund

Tao, Shengli 
Tateno, Ryunosuke

Taylor, Adam

Taylor, Jennifer

Tei, Shunsuke

Tejedor Vargas, Ernesto

Telling, Jennifer W.

Temesgena, Hailemariam

Temperli, Christian

Tenneson, Karis

Terzaghi, William

Tessier, Jack T.

Texier, Marion Le

Thévenon, Marie France

Thom, Dominik

Thomas, Barb

Thompson, Matthew P.

Thrippleton, Timothy

Thum, Tea

Tilman, Schenk

Tinya, Flóra

Tischer, Alexander

To, Kin-Ying

Toivonen, Johanna M.

Tomaštík, Julián

Tomiczak, Karolina

Tomter, Stein Michael

Tong, Tessie

Topalidou, Eleni

Torres Ruiz, José Manuel

Torres, Romina C.

Torresan, Chiara

Torres-Martínez, Lorena

Tramontini, Sara

Tremblay, Stéphane

Trentanovi, Giovanni

Tripathi, Abhishek Mani

Trocha, Lidia K.

Trømborg, Erik

Trouillier, Mario

Truong, Nguyen Le

Tsioras, Petros

Tsvetanov, Nickolay

Tucek, Jan

Tullis, Jason

Turner, Edgar

Turner, Mónica

Turner, Russell

Tyree, Michael

Tyukavina, Alexandra

Úbeda, Xavier

Ucar, Zennure

Uesugi, Akane
Uimari, Anne

Ujházyová, Mariana

Ulrich, Kristina

Umeki, Kiyoshi

Unger, Stephan

Upreti, Girish

Urban, Josef

Urbanski, Shawn P.

Urbinati, Carlo

Vackaru, Dava

Valade, Aude

Valbuena-Rabadán, Manuel Ángel

Valkonen, Sauli

Valls-Donderis, Pablo

Van Coillie, Frieke

Van De Gevel, Saskia

Van Der Maaten, Ernst

Van Der Sleen, Peter

Van Etten, Eddie J. B.

Vance, Eric

Vance, Marina

Vandenbussche, Filip

Vander Mijnsbrugge, Kristine

Vangansbeke, Pieter

Varner, Morgan

Vaughn, Nicholas

Vega, Flora Alonso

Vega-Nieva, Daniel

Velasco, Jordi Voltas

Venturas, Martin

Verdaguer, Dolors

Vetukuri, Ramesh

Vicente, Claudia

Vickers, Lance

Vidal, Alix

Vieira, Joana

Viennois, Gaëlle

Vijay, Varsha

Virzo De Santo, Amalia

Visser, Fleur

Vizzarri, Matteo

Vogt, Jess

Vokoun, Melinda

Volkova, Liubov

Vospernik, Sonja

Vourlitis, George L.

Vusic, Dinko

Vuts, Jozsef

Wagenbrenner, Joseph

Waldner, Peter

Walentowski, Helge

Walker, Xanthe 
Wallau, Marcelo

Waller, Donald

Wallin, Kimberly

Walters, Eric

Walters, Gretchen

Wang, Bin

Wang, Chi-Kuei

Wang, Jian

Wang, Jun

Wang, Yonghe

Wardlaw, Timothy

Warwell, Marcus V.

Watanabe, Atsushi

Watmough, Shaun

Weber, Norbert

Wei, Yu

Weidner, Helmut

Weiskittel, Aaron

Weissert, Lena

Welc, Monika

Wemheuer, Bernd

West, Thales A.P.

Westlind, Doug

White, Angela M.

White, Matthew

Whitfeld, Timothy

Wilkes-Allemann, Jerylee

Wilkin, Kate M.

Will, Rod

Williams, K. J. H

Williams, Marcus

Wills, Skye A.

Winkelstern, Ian

Winkler, Daniel E

Wojnicka-Półtorak, Aleksandra

Wolny, Elzbieta

Work, Courtney

Worth, James R.P.

Wright, William

Wulff, Sören

Xiao, Wen

Xie, Yingying
Xu, Li

Yamada, Yusuke

Yamori, Wataru

Yang, Wanqin

Yazaki, Tomotsugu

Yemshanov, Denys

Yen, Ting-Ming

Yetemen, Omer

Yi, Chuixiang

Yin, Chuntao

Yonenobu, Hitoshi

Young, Brian

Young, Timothy

Zaccone, Claudio

Zachara, Tadeusz

Zagajewski, Bogdan

Zaharia, Alina

Zambrano, Jenny

Zambrano-Wilson, Maria Clemencia

Zamora-Cristales, Rene

Zanetti, Michela

Zanin, Giampaolo

Zas, Rafael

Zatelli, Paolo

Zboril, Frantisek

Zborowska, Magdalena

Zdruli, Pandi

Zell, Jürgen

Zeller, Bernd

Zeng, Yelu

Zhai, Ruiting

Zhang, Jianwei

Zhang, Zhen

Zheng, Yuanrun

Ziaco, Emanuele

Zianis, Dimitris

Zimmerman, Barbara L.

Zimmerman, Jess

Živković, Vjekoslav

Zoglauer, Kurt

Zsedrovits, Tamás

(C) 2019 by the author. Licensee MDPI, Basel, Switzerland. This article is an open access article distributed under the terms and conditions of the Creative Commons Attribution (CC BY) license (http://creativecommons.org/licenses/by/4.0/). 